\title{
Editorial
}

\section{Antibodies Against M-Type Phospholipase Receptor and Prediction of Outcome in Membranous Nephropathy: We are Not There Yet}

\author{
Jack F.M. Wetzels \\ Department of Nephrology, Radboud University Medical Center, Nijmegen, The Netherlands
}

In 2009, Beck et al. [1] identified the M-type phospholipase receptor (PLA2R) as major antigen involved in membranous nephropathy. Antibodies against this protein, which is expressed on podocytes, were present in $70 \%$ of patients with idiopathic membranous nephropathy. The role of PLA2R was supported by a genome-wide association study that found an association between the $P L A 2 R$ gene and the development of membranous nephropathy. However, proof of the pathogenicity of PLA2Rantibodies (PLA2Rab) is lacking in the absence of an appropriate animal model (mice and rats do not express PLA2R on podocytes).

Still, the discovery of PLA2Rab and the subsequent development of a commercial qualitative immunofluorescence test (IFT) boasted clinical research. Many studies confirmed the presence of PLA2Rab in up to $80 \%$ of patients with membranous nephropathy. The presence of PLA2Rab is very specific for membranous nephropathy, thus allowing to diagnose membranous nephropathy without the need for kidney biopsy [2].

The development of quantitative ELISA assays (in house and commercial) stimulated studies that evaluated the role of antibody levels in predicting disease outcome.
The data were promising and indeed suggested that measurement of PLA2Rab might be of added value. Patients with high antibody levels at baseline were unlikely to develop spontaneous remission [3]. Similarly, in a study of rituximab treatment high antibody levels were associated with poor response [4]. Many studies provided support for the notion that PLA2Rab levels could predict outcome. Unfortunately, most studies were retrospective, biased, and of limited size.

Zhang et al. [5] evaluated the value of the presence and the level of PLA2Rab to predict disease outcome in a large patient cohort, who were followed up at Peking University first Hospital. The study included 359 patients, recruited in the period from January 2004 to December 2016. The authors conclude that both the presence as well as the level of PLA2Rab were independent predictors of remission. At first sight, these data derived from an unprecedented large patient cohort support implementation of quantitative measurement of PLA2Rab in clinical practice. However, a cautious note is needed, and early conclusions are not allowed. The manuscript clearly illustrates the difficulties of PLA2Rab predictor studies in membranous nephropathy.

\section{KARGER}

E-Mail karger@karger.com www.karger.com/ajn (c) 2018 The Author(s) Published by S. Karger AG, Basel

Karge

Open access

This article is licensed under the Creative Commons AttributionNonCommercial-NoDerivatives 4.0 International License (CC BYNC-ND) (http://www.karger.com/Services/OpenAccessLicense) Usage and distribution for commercial purposes as well as any distribution of modified material requires written permission.
Prof. Dr. Jack F.M. Wetzels

Department of Nephrology

Radboud University Medical Centre, 464

PO Box 9101, NL-6500 HB, Nijmegen (The Netherlands)

E-Mail jack.wetzels@ radboudumc.nl 


\section{How to Define PLA2R-Associated Membranous Nephropathy?}

For the comparison of outcome between patients with PLA2R-associated and not-PLA2R associated membranous nephropathy a gold standard test is needed. Zhang et al. [5] performed kidney biopsy staining to detect the PLA2R antigen and used commercially available IFT and ELISA tests. Of note, for the ELISA assay the authors have used a cut-off level of $20 \mathrm{RU} / \mathrm{mL}$ to define positivity. Out of 359 patients, 248 patients had positive biopsy staining. Of these, 195 patients were positive in IFT and ELISA, 39 were positive in IFT only, 7 were positive in ELISA only, and 7 were negative in both assays. If the kidney biopsy is the gold standard to diagnose PLA2R-associated membranous nephropathy, then IFT is the most sensitive test (94\%). The ELISA test is clearly less sensitive (81\%). Using the often-used cut-off value of $14 \mathrm{RU} / \mathrm{mL}$ did not improve sensitivity, with only 2 additional patients found positive. In a recent study, a cut-off value of $2.7 \mathrm{RU} / \mathrm{mL}$ was proposed [6]. Although a lower cut-off value may increase sensitivity, it will decrease specificity, and thus affect the use of the ELISA assay for diagnostic use. Studies are needed to determine if the combined use of IFT (to diagnose membranous nephropathy) and ELISA (to quantify PLA2Rab levels in IFT positive patients) has added value.

The use of the kidney biopsy as golden standard is debatable. Immunohistochemistry only confirms that the PLA2R-antigen is present in the immune deposits. Thus, kidney biopsy staining does not prove that PLA2Rab is present. Can we exclude that in some patients the presence of PLA2R might be an epiphenomenon, as has been shown for LIMP2 [7]. On the other hand, low staining intensities of PLA2R could be missed. Of note, in the study of Zhang et al. [5] immunofluorescence staining of IgG was absent in $15 \%$ of biopsies.

\section{How to Evaluate the Predictive Value of PLA2Rab Levels?}

Obviously, the predictive value of antibody levels can only be evaluated by using a quantitative assay (i.e., ELISA). Furthermore, the analysis should be limited to PLA2Rab-positive patients, and the independent role of antibody levels evaluated in multivariable regression analysis using anti-PLA2Rab levels as discontinuous (e.g., in quartiles) or continuous (preferably log-transformed) variable. In their study Zhang et al. [5] have used

Antibodies Against M-Type PLA2R and Outcome in Membranous Nephropathy
ELISA $<20 \mathrm{RU} / \mathrm{mL}$ as reference group. This invalidates the conclusions since the reference group thus includes both patients with PLA2R-associated membranous nephropathy with low antibody levels, as well as patients with non-PLA2R-associated membranous nephropathy.

\section{PLA2Rab as Predictor: Bias and Clinical Relevance}

To prevent biased conclusions and to assure that conclusions can be applied to clinical practice, studies must define and select the appropriate patient cohort. Zhang et al. [5] included patients who had received immunosuppressive therapy before sample collection. This introduced a bias. The prevalence of PLA2Rab was lower in these treated patients ( $47 \%$ vs. $69 \%$ ) suggesting that many patients had already developed immunological remission. The study also included 143 patients with non-nephrotic proteinuria. It is well known that patients with membranous nephropathy and non-nephrotic proteinuria have good outcome. We need predictors of outcome that can be used in patients with membranous nephropathy, normal renal function, and nephrotic syndrome. In a subgroup analysis Zhang et al. [5] indeed analysed risk factors for the outcome remission (an accepted surrogate end point in membranous nephropathy) in the subgroup of patients with membranous nephropathy, a nephrotic syndrome, not previously treated with immunosuppressive drugs. This subgroup included 173 patients. Unfortunately, the details of this subgroup are not provided. Still, in this subgroup, neither the presence nor the levels of PLA2Rab proved an independent predictive variable (see data in thier supplementary Table 9; for all online suppl material, see www.karger.com/doi/10.1159/000494661).

\section{Quantitative Analysis of PLA2Rab: An Ending or a New Beginning?}

It is important to realize that prediction of outcome is not 1 simple question. When considering immunosuppressive therapy in patients with membranous nephropathy there are 2 questions that are relevant: (1) What is the likelihood that my patient will develop a spontaneous remission? We know that approximately $40-50 \%$ of patients with membranous nephropathy will develop spontaneous remission and do not need immunosuppressive therapy. (2) What is the likelihood that my patient with membranous nephropathy and progres- 
sive disease will respond to immunosuppressive therapy? It is not unlikely that measurement of PLA2Rab might be relevant for only one of these questions. The data provided by Zhang et al. [5] do not allow solid conclusions. We would need additional information on the number of patients with nephrotic syndrome treated with immunosuppressive therapy, the timing of the start of therapy, and the period of follow-up. Most predictor studies of PLA2Rab have included patients who received immunosuppressive therapy. A recent study should be mentioned here. Van de Logt et al. [8] compared immunological remission rates in cohorts of patients treated with either rituximab or cyclophosphamide. There was no difference in immunological remission rate between these 2 treatments in patients with low or moderate antibody levels. In contrast, cyclophosphamide induced immunological remission in patients with high levels of antibodies, whereas rituximab was not effective. These data clearly suggest that measurement of PLA2Rab levels might be of great value, especially for the selection of immunosuppressive therapy. Well-developed prospective clinical studies are needed to validate these findings, to define the optimal cut-off levels, and assess sensitivity and specificity.

\section{Kidney Biopsies in the Era of PLA2Rab}

In another study from the same group of investigators, the role of pathological features in predicting treatment response and outcome was evaluated [9]. This study was largely based on the same patient cohort of consecutive patients with biopsy-proven membranous nephropathy. Somewhat unexplained, the number of patients was now 371 , although the study period was shorter (2008-2016). Other discrepancies are notable: median PLA2Rab level was lower (78 vs. $114 \mathrm{RU} / \mathrm{mL}$ ), and although follow-up (26.9 months) and the number of treated patients $(n=254)$ was similar, reported remission rates were quite different (complete remission 45 vs. $22 \%$ and partial remission 42 vs. 39\%). The authors observed that a higher stage of membranous nephropathy and a higher intensity of C3 staining were associated with a higher frequency of PLA2Rab positivity. There was however no association between PLA2Rab levels and pathological features. These data suggest that non-PLA2Rabassociated membranous nephropathy may indeed be less progressive, although it cannot be excluded that the absence of PLA2Rab in patients with stage I membranous nephropathy may simply reflect the situation of "the kidney as a sink", where all PLARab are absorbed in the kidney, and not noticeable in serum [10]. The authors should be able to provide a definite answer, since they have now stained all kidney biopsies for the PLA2R antigen. The authors next evaluated the role of pathological features in predicting remission or progressive kidney dysfunction. In multivariable analyses, no pathological feature was independently associated with remission, whereas PLA2Rab negativity was. Similarly, no remission and PLA2Rab positivity were independent predictors of progressive kidney dysfunction, whereas pathological features were not. Taken together, these data argue against the routine use of kidney biopsies in patients with PLA2Rab positive membranous nephropathy: the specificity of PLA2Rab is very high, thus a kidney biopsy is not needed to diagnose membranous nephropathy; apparently, since pathological features such as disease stage, C3-staining intensity, and tubule-interstitial inflammation do not predict outcome, a kidney biopsy also does not aid in clinical decision making.

\section{Disclosure Statement}

No conflicts of interest.

\section{Funding Source}

None.

\section{References}

1 Beck LH Jr, Bonegio RG, Lambeau G, Beck DM, Powell DW, Cummins TD, Klein JB, Salant DJ: M-type phospholipase A2 receptor as target antigen in idiopathic membranous nephropathy. N Engl J Med 2009;361: 11-21.

2 De Vriese AS, Glassock RJ, Nath KA, Sethi S, Fervenza FC: A proposal for a serology-based approach to membranous nephropathy. J Am Soc Nephrol 2017;28:421-430.

3 Hofstra JM, Debiec H, Short CD, Pellé T, Kleta R, Mathieson PW, Ronco P, Brenchley PE, Wetzels JF: Antiphospholipase A2 receptor antibody titer and subclass in idiopathic membranous nephropathy. J Am Soc Nephrol 2012;23:1735-1743.

\footnotetext{
4 Ruggenenti P, Debiec H, Ruggiero B, Chianca A, Pellé T, Gaspari F, Suardi F, Gagliardini E, Orisio S, Benigni A, Ronco P, Remuzzi G: Anti-phospholipase A2 receptor antibody titer predicts post-rituximab outcome of membranous nephropathy. J Am Soc Nephrol. 2015;26:25452558.
} 
5 QuZ, Zhang M, Cui Z, WangJ, Wang M, Zhang Y, Wang F, Wang X, Meng L, Cheng X, Liu G, Zhao M: Antibodies against M-type phospholipase A2 receptor may predict treatment response and outcome in membranous nephropathy. Am J Nephrol 2018;48:438-446.

6 Tampoia M, Migliucci F, Villani C, Abbracciavento L, Rossini M, Fumarulo R, Gesualdo L, Montinaro V: Definition of a new cut-off for the anti-phospholipase A2 receptor (PLA2R) autoantibody immunoassay in patients affected by idiopathic membranous nephropathy. J Nephrol 2018, Epub ahead of print.
7 Rood IM, Merchant ML, Wilkey DW, Zhang T, Zabrouskov V, van der Vlag J, Dijkman HB, Willemsen BK, Wetzels JF, Klein JB, Deegens JK: Increased expression of lysosome membrane protein 2 in glomeruli of patients with idiopathic membranous nephropathy. Proteomics 2015;15:3722-3730.

8 Van de Logt AE, Dahan K, Rousseau A, van der Molen R, Debiec H, Ronco P, Wetzels J: Immunological remission in PLA2R-antibody-associated membranous nephropathy: cyclophosphamide versus rituximab. Kidney Int 2018;93:1016-1017.
9 Zhang XD, Cui Z, Zhang MF, Wang J, Zhang YM, Qu Z, Wang X, Huang J, Wang F, Meng LQ, Cheng XY, Wang SX, Liu G, Zhao MH: Clinical implications of pathological features of primary membranous nephropathy. BMC Nephrol 2018;19:215.

10 Van de Logt AE, Hofstra JM, Wetzels JF: Serum anti-PLA2R antibodies can be initially absent in idiopathic membranous nephropathy: seroconversion after prolonged follow-up. Kidney Int 2015;87:1263-1264. 